\title{
DIFFERENTIATION WITH RESPECT TO $\phi$-PSEUDO-STRONG BLANKETS AND RELATED PROBLEMS
}

\section{CHARLES A. HAYES}

1. Introduction. In a paper by A. P. Morse, ${ }^{1}$ there is developed a rather complete theory of differentiation, a substantial part of which is concerned with the differentiation of certain set functions with respect to strong blankets. ${ }^{2}$ It is the purpose of the present paper to show that a satisfactory theory of differentiation still holds when the hypotheses on the blanket are relaxed considerably, while retaining much of Morse's method. More specifically, we shall show that additive set functions of a quite general type are differentiable with respect to blankets whose covering families are not disjointed, but whose overlapping is suitably controlled; at the same time, it is pointed out by an example that this control cannot be relaxed much further without a distinct loss in differentiability properties. We conclude the paper with some remarks concerning the unions of certain blankets.

2. Preliminaries. We shall find it convenient to adopt much of the terminology of Morse's paper, mentioned above. However, since his terminology is not widely used, it will be desirable to define these terms. We, therefore, state at this point a number of conventions and definitions which we shall require.

Throughout this paper we shall let $\mathfrak{\subseteq}$ denote a fixed space metrized by the fixed function $\rho$, so that

$$
0=\rho(x, x) \leqq \rho(x, y)=\rho(y, x) \leqq \rho(x, z)+\rho(y, z)<\infty
$$

whenever $x, y$, and $z$ are in $\Im$. Such terms as "distance," "Borel," "closed," etc., will have reference to $\rho$, and will be used with their usual meanings. In particular, we shall agree to write "diam $A$ " in place of "the diameter of $A$ " whenever $A \subset \mathfrak{S}$; we further agree that $\operatorname{diam} 0=0$.

If $\mathfrak{F}$ is any family, we shall let $\sigma \mathfrak{F}$ denote the set of those points $x$ for which there exists some $\beta \in \mathfrak{F}$ such that $x \in \beta$.

If $A$ and $B$ are any two sets, we let $A-B$ denote the set of those points which are in $A$ and not in $B$. If $A \subset \mathfrak{S}$, we define $\tilde{A}=\Im-A$.

Presented to the Society, December 28, 1951; received by the editors May 15, 1951.

1 See A. P. Morse, $A$ theory of covering and differentiation, Trans. Amer. Math. Soc. vol. 55 (1944) pp. 205-235.

${ }^{2}$ Unfamiliar technical terms will be defined in $\$ \$ 2,5$, and 7 . 
We agree to let $S(x)$ denote the set whose sole member is $x$.

If $B$ is any family of subsets of $\mathfrak{S}$, and $x \in \mathfrak{S}$, then we shall henceforth let $P_{\&}(x)$ denote the number of members of (s) to which $x$ belongs. It is clear that if $(S)$ is a countable family of Borel subsets of $\mathfrak{S}$, then for each $\phi \in \mathfrak{B},{ }^{3} P_{\mathscr{S}}$ is a $\phi$-measurable function.

2.1. Definition. $\phi$ measures $\subseteq$ if and only if $\phi$ is such a function that

$$
\phi(A) \leqq \sum_{\beta \in \mathfrak{F}} \phi(\beta)
$$

whenever $\mathfrak{F}$ is a countable family and $A \subset \sigma \mathfrak{F} \subset \mathfrak{S}$.

2.2. Definition. We define $\mathfrak{U}$ as that family for which $\phi \in \mathfrak{U}$ if and only if $\phi$ measures $\mathfrak{S}$, and in addition

$$
\phi(A)+\phi(B)=\phi(A+B),
$$

whenever $A$ and $B$ are subsets of $\subseteq$ which are at a positive distance apart.

2.3. Definition.

$$
\mathfrak{B}=\mathfrak{U} \cdot \underset{\phi}{E}[\phi(\beta)<\infty \text { for each bounded set } \beta \subset \mathbb{S}] \text {. }
$$

2.4. Definition. $A$ is $\phi$ measurable if and only if $\phi$ measures $\subseteq$ and

$$
\phi(T)=\phi(T A)+\phi(T \tilde{A})
$$

for each subset $T$ of $\subseteq$.

2.5. Definition. The limit notations

$$
\underset{\mathfrak{F} \ni \beta \rightarrow x}{\operatorname{limalinf}} f(\beta), \quad \underset{\mathfrak{F} \ni \beta \rightarrow x}{\operatorname{limalsup}} f(\beta),
$$

in which $\mathfrak{F}$ is a family of subsets of $\mathfrak{S}$, and $x \in \mathfrak{S}$, are defined, respectively, as

$$
\lim _{t \rightarrow 0+}\left\{\inf _{\beta \in H(t)} f(\beta)\right\}, \quad \lim _{t \rightarrow 0+}\left\{\sup _{\beta \in H(t)} f(\beta)\right\}
$$

where, for $t>0$,

$$
H(t)=\mathfrak{F} \cdot \underset{\beta}{E}[\operatorname{diam}(\beta+S(x))<t] .
$$

We shall write

$$
\operatorname{limal}_{\mathfrak{F} \rightarrow \beta \rightarrow x} f(\beta)=\operatorname{limalsup}_{\Re \ni \beta \rightarrow x} f(\beta)
$$

${ }^{3}$ For a definition of $\mathfrak{B}$, see $2.1,2.2$, and 2.3 . 
if and only if

$$
\operatorname{limalsup}_{\mathfrak{F} \ni \beta \rightarrow x} f(\beta)=\operatorname{limalinf}_{\mathfrak{F} \ni \beta \rightarrow x} f(\beta) .
$$

2.6. Definition. We say that $\mathfrak{F}$ covers $\phi$-almost all of $A$ if and only if $\phi \in \mathfrak{B}$ and $\mathfrak{F}$ is a family for which $\phi(A-\sigma \mathfrak{F})=0$.

2.7. Definition. We say that $F$ is a blanket if and only if $F$ is such a function that for $x$ in its domain:

(i) $x \in \mathfrak{S}$ and $F(x)$ is a nonvacuous family of subsets of $\mathfrak{S}$;

(ii) $\operatorname{diam} \beta<\infty$ whenever $\beta \in F(x)$;

(iii) $\inf _{\beta \in F(x)}\{\operatorname{diam}(\beta+S(x))\}=0$.

2.8. Definition. If $F$ is a blanket with domain $A$, then the set

$$
\sum_{x \in A} F(x)
$$

is called the spread of $F$.

2.9. Definition. $F$ is a Borelish (close) blanket if and only if $F$ is such a blanket that $\beta \in F(x)$ implies $\beta$ is a Borel (closed) set.

2.10. Definition. $F$ is a $\phi$-heavy blanket if and only if $\phi \in \mathfrak{B}, F$ is a Borelish blanket with domain $A$, and there exists in the spread of $F$ a countable disjointed family which covers $\phi$-almost all of $A$.

2.11. Definition. $G$ is a subblanket of $F$ if and only if $F$ and $G$ are such blankets that $G(x) \subset F(x)$ whenever $x$ is in the domain of $G$.

2.12. Definition. $F$ is a $\phi$-strong blanket if and only if $F$ is a blanket each of whose subblankets is $\phi$-heavy.

2.13. Definition. $F \odot \beta$ is the family for which $G \in(F \odot \beta)$ if and only if $G$ is a subblanket of $F$ whose domain is the intersection of $\beta$ with the domain of $F$.

2.14. Definition.

$$
\begin{gathered}
D^{*}(f, g, F, x)=\operatorname{limalsup}_{F(x) \ni \beta \rightarrow x} \frac{f(\beta)}{g(\beta)} ; \\
D_{*}(f, g, F, x)=\operatorname{limalinf}_{F(x) \ni \beta \rightarrow x} \frac{f(\beta)}{g(\beta)} ; \quad D(f, g, F, x)=\operatorname{limal}_{F(x) \ni \beta \rightarrow x} \frac{f\left(\beta^{*}\right)}{g(\beta)} .
\end{gathered}
$$

For the remainder of this paper, we shall let $\phi$ denote an arbitrary fixed member of the 2.3 set $\mathfrak{B}$. The following theorems of $\$ \$ 3$ and 4 are needed for later application.

3. Construction of certain measures. Throughout this section we shall let $f$ be a non-negative real valued function whose domain $\mathfrak{M}$ is a family of subsets of $\mathfrak{S}$.

3.1. Definition. For each $\epsilon>0$ and each $s \subset \mathcal{S}$, we let $\Gamma(f, \epsilon, s)$ de- 
note the inf of numbers of the form

$$
\sum_{\beta \in \Theta} f(\beta),
$$

where $\mathbb{B}$ is such a countable subfamily of $\mathfrak{M}$ that $\phi(s-\sigma(\mathfrak{S})<\epsilon$.

3.2. Definition. We define $f^{\nabla}$ as that function on

$$
E[s \subset \mathfrak{s}]
$$

for which

$$
f^{\nabla}(s)=\lim _{\epsilon \rightarrow 0+} \Gamma(f, \epsilon, s)
$$

whenever $s \subset \mathfrak{S}$.

If we keep in mind that inf $0=\infty$, it is clear that $0 \leqq \Gamma(f, \epsilon, s) \leqq \infty$ whenever $\epsilon>0$ and $s \subset \mathfrak{S}$; furthermore, $\Gamma(f, \epsilon, s)$ increases as $\epsilon$ tends towards zero, so that $0 \leqq f^{\nabla}(s) \leqq \infty$ whenever $s \subset \mathfrak{S}$. Also, $A \subset B \subset \subseteq$ implies $f^{\nabla}(A) \leqq f^{\nabla}(B)$. From these facts and the above definitions, the proofs of the following three simple lemmas may easily be established.

3.3. LemmA. If $s \subset \Im, \epsilon>0$, and $N$ is such a finite number that $0 \leqq N \leqq f^{\nabla}(s)$, then there exists such a positive number $\alpha<\epsilon$ that if (s) is any countable subfamily of $\mathfrak{M}$ for which $\phi(s-\sigma(\mathfrak{S})<\alpha$, then

$$
N \leqq \sum_{\beta \in \Theta} f(\beta)+\epsilon \text {. }
$$

3.4. Lemma. If $\epsilon>0, s \subset \subseteq$, and $f^{\nabla}(s)<\infty$, then there exists such $a$ countable family $\mathbb{M} \subset \mathfrak{M}$ that

$$
\phi\left(s-\sigma(S)<\epsilon ; \quad \sum_{\beta \in \Theta} f(\beta) \leqq f^{\nabla}(s)+\epsilon .\right.
$$

3.5. Lemma. (1) If $\beta \in \mathfrak{M}$, then $f^{\nabla}(\beta) \leqq f(\beta)$;

(2) $\phi(s)=0$ implies $f^{\nabla}(s)=0$.

3.6. TheOREM. $f^{\nabla}$ measures $\mathfrak{S}$.

Proof. With 2.1 in mind, we let $\mathfrak{S}$ denote an arbitrary countable family of subsets of $\subseteq$, choose an arbitrary positive number $\epsilon$, and let $\eta$ denote a positive real valued function for which

$$
\sum_{\beta \in \mathfrak{S}} \eta_{\beta}<\epsilon .
$$

It has to be shown that

$$
f^{\nabla}(\sigma \mathfrak{S}) \leqq \sum_{\beta \in \mathfrak{W}} f^{\nabla}(\beta)
$$


We discard the trivial case where the right side of this inequality is infinite; we accordingly assume it is finite. Thus we may apply Lemma 3.4 to determine for each $\beta \in \mathfrak{S}$ such a countable family $\mathfrak{B}_{\beta} \subset \mathfrak{M}$ that

$$
\begin{gathered}
\phi\left(\beta-\sigma \mathfrak{S}_{\beta}\right)<\eta_{\beta} ; \\
\sum_{t \in \mathscr{\bigotimes}_{\beta}} f(t) \leqq f^{\nabla}(\beta)+\eta_{\beta} .
\end{gathered}
$$

We let $\Omega=\sum_{\beta} \in \mathfrak{S}\left(_{\beta}\right.$, note that $\Omega$ is a countable subfamily of $\mathfrak{M}$, check that

$$
\begin{aligned}
\phi(\sigma \mathfrak{S}-\sigma \mathfrak{R}) & \leqq \sum_{\beta \in \mathfrak{W}} \phi\left(\beta-\sigma\left(\mathfrak{S}_{\beta}\right)<\sum_{\beta \in \mathfrak{W}} \eta_{\beta}<\epsilon,\right. \\
\sum_{t \in \mathfrak{\Omega}} f(t) & \leqq \sum_{\beta}\left(\sum_{t \in \mathfrak{W}} f(t)\right) \leqq \sum_{\beta}\left(f^{\nabla}(\beta)+\eta_{\beta}\right)<\sum_{\beta} f_{\mathfrak{S}} f^{\nabla}(\beta)+\epsilon,
\end{aligned}
$$

and thus conclude that

$$
\Gamma(f, \epsilon, \sigma \mathfrak{S}) \leqq \sum_{\beta \in \mathfrak{S}} f^{\nabla}(\beta)+\epsilon ;
$$

since $\epsilon$ is arbitrary, we see that (1) holds; thus $f^{\nabla}$ measures $\subseteq$.

4. Some properties of measures belonging to $\mathfrak{B}$. We recall the definitions of $P_{\mathbb{S}}(x)$ and $S(x)$ as given in the remarks preceding 2.1.

4.1. TheOREM. If $\mathbb{H}$ is a countable family of Borel subsets of $\mathfrak{S}$ and $\psi \in \mathfrak{B}$, then

$$
\sum_{t \in \Theta} \psi(t)=\int_{\sigma \Theta} P_{\Theta}(x) d \psi(x)
$$

Proof. That the theorem holds for any finite family of Borel sets may be shown by a straightforward induction. Next, we consider a countably infinite family $\mathfrak{B}$ of Borel sets. We let $B$ and $\mathfrak{S}$ be such sequences that

$$
\text { (S) }=\sum_{i=1}^{\infty} \mathbf{S}\left(B_{i}\right), \quad \mathfrak{W}_{k}=\sum_{i=1}^{k} \mathbf{S}\left(B_{i}\right)
$$

for each positive integer $k$. From the monotone nature of the sequence $\mathfrak{S}$ and the fact that $\mathfrak{W}_{k}$ is a finite family for $k=1,2,3, \cdots$ we obtain the desired result, namely,

$$
\sum_{t \in \mathscr{S}} \psi(t)=\lim _{k \rightarrow \infty} \sum_{t \in \mathfrak{\Phi}_{k}} \psi(t)=\lim _{k \rightarrow \infty} \int_{\sigma \mathfrak{\Phi}_{k}} P_{\mathfrak{\$}_{k}}(x) d \psi(x)=\int_{\sigma \infty} P(x) d \psi(x) .
$$

4.2. Theorem. If $F$ is a Borelish blanket, $\beta$ is a bounded Borel set, 
$\psi \in \mathfrak{B}$, and $\epsilon>0$, then there exists a bounded open set $\alpha \supset \beta$ and an $F^{\prime} \in(F \odot \alpha)$ for which

$$
\sum_{B \in \Theta} \psi(B) \leqq \psi(\beta)+\epsilon
$$

whenever (S) is such a countable subfamily of the spread of $F^{\prime}$ that

$$
\int_{\sigma \leftrightarrow 3}\left(P_{\Theta}(x)-1\right) d \psi(x) \leqq \epsilon / 2 \text {. }
$$

Proof. We determine a bounded open $\operatorname{set}^{4} \alpha \supset \beta$ for which

$$
\psi(\alpha) \leqq \psi(\beta)+\epsilon / 2,
$$

let $A$ denote the domain of $F$, so define $F^{\prime}$ that for each $x \in A \cdot \alpha$,

$$
F^{\prime}(x)=F(x) \cdot \underset{t}{E}[t \subset \alpha]
$$

recall Definition 2.13 to check that $F^{\prime} \in(F \odot \alpha)$, and select any countable family $\&$ from the spread of $F^{\prime}$ for which

$$
\int_{\sigma \mathbb{S}}\left(P_{\mathbb{S}}(x)-1\right) d \psi(x) \leqq \epsilon / 2 .
$$

Using (1), (2), Theorem 4.1, and the fact that $\sigma \leftrightarrow \subset \alpha$, we see that

$$
\begin{aligned}
\sum_{B \in \mathcal{S}} \psi(B) & =\int_{\sigma \Theta} P_{\mathscr{S}}(x) d \psi(x) \\
& \leqq \int_{\sigma \in \mathcal{S}}\left(P_{\mathcal{S}}(x)-1\right) d \psi(x)+\psi(\alpha) \leqq \psi(\beta)+\epsilon,
\end{aligned}
$$

which was to be proved.

\section{Differentiation with respect to a $\phi$-pseudo-strong blanket.}

5.1. Definition. We say that $F$ is a $\phi$-pseudo-heavy blanket if and only if $F$ is a Borelish blanket with domain $A$, and corresponding to each $\epsilon>0$ and $e a c h^{5} \psi \in \mathfrak{B}$, there exists in the spread of $F$ a countable family (S) for which

(i) $\phi(A-\sigma$ (S) $<\epsilon$;

(ii) $\int_{\sigma \leftrightarrow}\left(P_{\leftrightarrow}(x)-1\right) d \psi(x)<\epsilon$.

5.2. Definition. We say that $F$ is a $\phi$-pseudo-strong blanket if

${ }^{4}$ That such an open set exists whenever $\psi \in \mathfrak{B}$ and $\beta$ is a bounded Borel set follows easily from the article of A. P. Morse, The role of internal families in measure theory, Bull. Amer. Math. Soc. vol. 50 (1944) pp. 723-728.

5 The importance of this requirement is brought out in Part II of $\S 6$. 
and only if $F$ is a blanket each of whose subblankets is $\phi$-pseudo-heavy.

Throughout this section we shall let $F$ denote an arbitrary fixed $\phi$-pseudo-strong blanket with domain $A$ and spread $\mathfrak{F}$.

Referring to Definition 2.12, we see that each $\phi$-strong blanket is $\phi$-pseudo-strong. That the converse is not true can be shown by an example which is given in $\$ 6$. Condition 5.1 (ii) thus allows a certain degree of overlapping in the covering subfamilies of $\mathfrak{F}{ }^{6}$

For each $\psi \in \mathfrak{B}$, we find it desirable at times to restrict the domain of $\psi$ to the family $\mathfrak{F}$; we let $\psi_{\mathfrak{F}}$ denote the so restricted function. Recalling Definitions 3.1 and 3.2, we further so define $\Psi$ that for each $s \subset \mathfrak{S}$,

$$
\bar{\psi}(s)=\lim _{\epsilon \rightarrow 0+} \Gamma\left(\psi_{\mathfrak{F}}, \epsilon, s\right) .
$$

Of the following two lemmas, the first is a direct consequence of Theorem 3.6, and the second is a result of the definition of $\Phi$ and Lemma 3.5, (2).

5.3. Lemma. If $\psi \in \mathfrak{B}$, then $\bar{\psi}$ measures $\mathfrak{S}$.

5.4. LEMMA. (1) $\phi(s) \leqq \Phi(s)$ whenever $s \subset \subseteq$;

(2) $\phi(s)=0$ is equivalent to $\phi(s)=0$.

5.5. TheOREM. If $\psi \in \mathfrak{B}, \epsilon>0, F^{\prime}$ is a subblanket of $F$ with domain $B$ and spread $\mathfrak{F}^{\prime}$, then $\mathfrak{F}^{\prime}$ contains a countable family $\mathbb{F}$ for which

$$
\phi\left(B-\sigma(S)<\epsilon ; \quad \sum_{t \in \circlearrowleft} \psi(t) \leqq \bar{\psi}(B)+\epsilon .\right.
$$

Proof. We may assume that $\Psi(B)<\infty$, since otherwise the theorem is true due to the $\phi$-pseudo-strength of $F$. We use Lemma 3.4 to determine a countable family $\mathfrak{S} \subset \mathfrak{F}$ for which

$$
\phi(B-\sigma \mathfrak{S})<\frac{\epsilon}{2} ; \quad \sum_{\beta \in \mathfrak{W}} \psi(\beta)=\sum_{\beta \in \mathfrak{W}} \psi_{\mathfrak{F}}(\beta) \leqq \bar{\psi}(B)+\frac{\epsilon}{2} .
$$

We next select such a positive real valued function $\eta$ that

${ }^{6} \mathrm{C}$. Y. Pauc thought of, and through his colleague O. K. Househam suggested to the writer, the possibility of measuring overlap by an integral of the kind mentioned in condition (I), Part II, $\$ 6$ of this paper. Other measures of overlap are considered by O. Haupt and C. Y. Pauc in their papers, Vitalische Systeme in Booleschen SigmaVerbanden, Sitzungsberichte der Mathematisch-Naturwissenschaftlichen Klasse der Bayerischen Akademie der Wissenschaften zu München (1950) pp. 187-207; and, Contributions to the abstract theory of differentiation of set and soma functions, to appear in Proc. Cambridge Philos. Soc. Pauc states that these papers were inspired by R. de Possel, Derivation abstrait des fonctions d'ensemble, J. Math. Pures Appl. (1936) pp. 391-409. 


$$
\sum_{\beta \in \mathfrak{S}} \eta_{\beta}<\frac{\epsilon}{2}
$$

invoke Theorem 4.2 and the $\phi$-pseudo-strength of $F$ to determine for each $\beta \in \mathfrak{W}$ an open set $\alpha_{\beta}$ and a countable family $\Omega_{\beta} \subset \mathfrak{F}^{\prime}$ for which

$$
\beta \subset \alpha_{\beta}, \quad \phi\left(B \alpha_{\beta}-\sigma \Re_{\beta}\right)<\frac{\eta_{\beta}}{2}, \quad \sum_{t \in \Re_{\beta}} \psi(t) \leqq \psi(\beta)+\eta_{\beta},
$$

let $\mathfrak{S}=\sum_{\beta \in \mathfrak{F}} \Omega_{\beta}, a=\sum_{\beta \in \mathfrak{F}} \alpha_{\beta}$, note that $\mathbb{B}$ is a countable subfamily of $\mathfrak{F}^{\prime}$, and employ (1) and (2) to see that

$$
\begin{gathered}
\phi\left(B-\sigma(\mathfrak{S}) \leqq \phi(B-\sigma \mathfrak{W})+\phi(\sigma \mathfrak{S}-a)+\sum_{\beta \in \mathfrak{W}} \phi\left(B \alpha_{\beta}-\sigma \Re_{\beta}\right)<\epsilon ;\right. \\
\sum_{t \in \mathcal{G}} \psi(t) \leqq \sum_{\beta \in \mathfrak{S}}\left(\sum_{t \in \mathfrak{R}_{\beta}} \psi(t)\right) \leqq \sum_{\beta \in \mathfrak{S}}\left(\psi(\beta)+\eta_{\beta}\right) \leqq \bar{\psi}(B)+\epsilon .
\end{gathered}
$$

5.6. ThEOREM. If $0<\lambda<\infty, B \subset \mathfrak{S}, F^{\prime}$ is a subblanket of $F, g$ and $h$ are members of $\mathfrak{B}$, and

$$
\operatorname{limal}_{F^{\prime}(x) \ni \beta \rightarrow x} \frac{g(\beta)}{h(\beta)}<\lambda
$$

for each $x \in B$, then $\bar{g}(B) \leqq \lambda \bar{h}(B)$.

Proof. We so define $F^{\prime \prime}$ that for each $x \in B$,

$$
F^{\prime \prime}(x)=F^{\prime}(x) \cdot E_{8}[g(s)<\lambda h(s)] .
$$

Our hypotheses insure that $F^{\prime \prime}$ is a subblanket of $F$ with domain $B$. We selet $\epsilon>0$, let $N$ be any finite number for which $0 \leqq N \leqq \bar{g}(B)$, and invoke Lemma 3.3 and Theorem 5.5 to determine a number $\alpha$ and a countable family $\mathfrak{S}$ in the spread of $F^{\prime \prime}$ for which

$$
\begin{gathered}
0<\alpha<\frac{\epsilon}{(\lambda+1)}, \quad \phi(B-\sigma \mathfrak{S})<\alpha \\
\sum_{\beta \in \mathfrak{W}} h(\beta) \leqq \bar{h}(B)+\alpha, \quad N \leqq \sum_{\beta \in \mathfrak{W}} g(\beta)+\frac{\epsilon}{(\lambda+1)} .
\end{gathered}
$$

From (1) and (2) we obtain

$$
N \leqq \sum_{\beta \in \mathfrak{S}} g(\beta)+\frac{\epsilon}{(\lambda+1)} \leqq \lambda \sum_{\beta \in \mathfrak{S}} h(\beta)+\frac{\epsilon}{(\lambda+1)} \leqq \lambda \bar{h}(B)+\epsilon,
$$

and this inequality leads at once to the required result. 
From this point on, the theory parallels almost exactly the various parts of Morse's Theorem $8.5 ;^{7}$ the overlapping of the covering families causes no difficulty. Therefore we merely state the final result.

5.7. TheOREM. If $\psi \in \mathfrak{B}$, then $0 \leqq D(\psi, \phi, F, x)<\infty$ for $\phi$-almost all $x \in A$.

5.8. REMARK. A class of blankets which contains, but is distinct from, the class of all $\phi$-strong blankets, and which is itself a proper subset of the class of $\phi$-pseudo-strong blankets, is obtained by replacing, in Definitions 5.1 and 5.2, condition 5.1 (ii) by the statement: (ii) $^{\prime}(5)$ is disjointed. With practically no changes in the theory just developed, we can show that if $G$ is a blanket of this new type, and $f$ is $\phi$-addivelous, ${ }^{8}$ then $0 \leqq D(f, \phi, G, x)<\infty$ for $\phi$-almost all $x$ in the domain of $G$. This is a slight generalization of Morse's Theorem 8.5.

6. Two constructions. Throughout this section we let $\Re_{2}(=\mathbb{S})$ denote the euclidean plane, which is assumed to be represented by cartesian coordinates, and we let $L(=\phi)$ represent plane Lebesgue measure. We denote by $S$ the open unit square in $\Re_{2}$ with principal vertices at $(0,0)$ and $(1,1)$. We also let $n$ and $t$ denote, respectively, an arbitrary positive integer and an arbitrary point of $S$.

Part I. We now construct a blanket which is $L$-pseudo-strong but not $L$-strong. For this purpose, we let $I_{n, t}, \dot{I}_{n, t}$, and $\ddot{I}_{n, t}$ designate, respectively, closed squares centered at $t$, with sides parallel to the axes and of length $2^{-n}, 3 \cdot 2^{-n}$, and $9 \cdot 2^{-n}$. For each point $(x, y)=t$ we let $K_{t}$ denote the set of points in $\Re_{2}$ of the form $(x+r, y+s)$, where $r$ and $s$ are arbitrary rational numbers. Finally, we define $J_{n, t}=I_{n, t}$ $+K_{t} \cdot \ddot{I}_{n, t}$. We shall call $I_{n, t}$ the set of inner points of $J_{n, t}$, and $J_{n, t} \cdot I_{n, t}$ the set of outer points of $J_{n, t}$.

To each set $\beta$ of the form $\beta=J_{n, t}$, there correspond uniquely the sets $\beta^{\prime}=I_{n, t}$ and $\beta^{\prime \prime}=\dot{I}_{n, t}$, and conversely. Whenever we speak of corresponding sets in the work at hand it is to be understood in this sense. It is easy to see that

(1) $L(\beta)=L\left(\beta^{\prime}\right)=(1 / 9) L\left(\beta^{\prime \prime}\right)$;

(2) if $\beta_{1} \cdot \beta_{2}=0$, then $\beta_{1}^{\prime} \cdot \beta_{2}^{\prime}=\beta_{1}^{\prime \prime} \cdot \beta_{2}^{\prime \prime}=0$.

We define $T$ as that blanket with domain $S$ for which

$$
T(t)=\sum_{n=1}^{\infty} S\left(J_{n, t}\right)
$$

We assert that $T$ is not $L$-strong. To show this, we take a sub-

${ }^{7}$ See footnote 1.

${ }^{8}$ See Definition 8.3 of Morse's paper on differentiation. 
blanket of $T$, say $T_{0}$, with domain $S$, each member of whose spread is contained in $S$, and pick an arbitrary countable disjointed subfamily (S) of this spread. We let $\left(\mathcal{S}^{\prime \prime}=\sum_{\beta \in \mathbb{G}} \beta^{\prime \prime}\right.$. We note that $\sigma \mathcal{B}^{\prime \prime} \subset S$, use (1) and (2), and obtain $L(S-\sigma()) \geqq L(S)-\sum_{\beta \in \mathcal{G}} L(\beta)=1-(1 / g)$. $\sum_{\beta \in \Theta} L\left(\beta^{\prime \prime}\right)=1-(1 / 9) L\left(\sigma \mathcal{S}^{\prime \prime}\right) \geqq 8 / 9$. Hence $T$ is not $L$-strong.

We further declare that $T$ is $L$-pseudo-strong. To show this, we first select an arbitrary member $\psi$ of the set $\mathfrak{B}$, let $M$ denote the set of points $u \in \Re_{2}$ for which $\psi(S(u))>0$, and define $N$ as the set of points of the form $(x+r, y+s)$, where $r$ and $s$ are arbitrary rational numbers and $(x, y) \in M$. We choose an arbitrary subblanket of $T$, say $T_{1}$, with domain $S_{1} \subset(S-N)$. Since $N$ is countable, making $L(N)=0$, the $L$-pseudo-strength of $T$ will be established if we can find in the spread of $T_{1}$ a countable family (S) for which

$$
L\left(S_{1}-\sigma(3)=0, \quad \int_{\sigma \leftrightarrow}\left(P_{\Theta}(u)-1\right) d \psi(u)=0 .\right.
$$

We so define the blanket $U_{1}$ that for $t \in S_{1}$,

$$
U_{1}(t)=\sum_{\beta \in T_{1}(t)} S\left(\beta^{\prime}\right),
$$

and use the obvious $L$-strength of $U_{1}$ to find a countable disjointed subfamily $\mathfrak{S}$ of the spread of $U_{1}$ for which $L\left(S_{1}-\sigma \mathfrak{E}\right)=0$. There exists a corresponding countable family (not necessarily disjointed)

$$
(S)=\sum_{\beta^{\prime} \in \mathfrak{S}} S(\beta)
$$

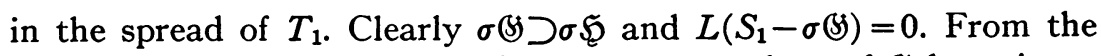
disjointedness of $\mathfrak{S}$ we know that no two members of $\$ S$ have inner points in common, so that the set of those points $u \in \sigma(S)$ for which $P_{\Theta}(u)>1$ must lie in the countable set $Q$ which embraces all outer points of members of $\mathfrak{S}$. However, the centers of the members of $\mathfrak{S}$ cannot be points of $N$, so that the outer points of members of $(B)$ cannot be points of $M$; thus $\psi(S(u))=0$ for each $u \in Q$. Therefore,

$$
\int_{\sigma \Theta}\left(P_{\Theta}(u)-1\right) d \psi(u) \leqq \int_{Q}\left(P_{\Theta}(u)-1\right) d \psi(u)=0,
$$

and $T$ is $L$-pseudo-strong.

Part II. We wish to show that the control exercised upon the overlapping in 5.1 (ii) cannot be relaxed much without a distinct loss in differentiability properties of our blankets. For this purpose, we consider a blanket $H$ having the property that given any $\epsilon>0$ and any subblanket $H^{\prime}$ with domain $A^{\prime}$, then there exists in the spread of 
$H^{\prime}$ a countable family $\mathfrak{E}$ for which

$$
\int_{\sigma \mathfrak{S}}\left(P_{\mathfrak{S}}(x)-1\right) d \phi(x)<\epsilon ; \quad \phi\left(A^{\prime}-\sigma \mathfrak{W}\right)=0 .
$$

The question arises: if $\psi \in \mathfrak{B}$, must it be true that $0 \leqq D(\psi, \phi, H, x)$ $<\infty$ for $\phi$-almost all $x$ in the domain of $H$ ? We shall show, by an example in $\Re_{2}$, that the answer is negative even when $\psi$ is an $L$-absolutely continuous set function of a very special kind.

We define $\mathfrak{M}_{n}$ as the set of all points in $\Re_{2}$ of the form $\left(p / 2^{n^{2}}, q / 2^{n^{2}}\right)$ where $p$ and $q$ are arbitrary integers; we define $\mathfrak{B}_{n}$ as the family of all closed squares whose centers are points of $\mathfrak{M}_{n}$, with sides parallel to the axes and of length $n^{-1} \cdot 2^{-\left(n^{2}+n\right)}$.

We so define the sequence $f$ of Borel functions, and $g$, that

$$
\begin{aligned}
& f_{n}(u)=n^{3} \quad \text { if } \quad u \in S \cdot \sigma \mathfrak{B}_{n}, \quad f_{n}(u)=0 \quad \text { if } \quad u \in\left(\Re_{2}-S \cdot \sigma \mathfrak{B}_{n}\right) \text {; } \\
& g(u)=\sum_{k=1}^{\infty} f_{k}(u) \quad \text { for each } u \in \Re_{2} ;
\end{aligned}
$$

we further so define $\Omega$ and $\Omega^{\prime}$ that for each Borel set $\beta \subset \Re_{2}$,

$$
\Omega(\beta)=\int_{\beta} g(u) d L(u),
$$

and for each set $s \subset \Re_{2}$,

$$
\Omega^{\prime}(s)=\inf _{\beta \in B_{s}} \Omega(\beta),
$$

where $B_{8}$ is the family of all Borel subsets of $\Re_{2}$ which contain $s$.

\subsection{Theorem. If $p \geqq 1$, then $g \in L^{(p)}$.}

PROof. There are $\left(2^{n^{2}}+1\right)^{2}$ points of $\mathfrak{M}_{n}$ lying in the closure of $S$, each the center of a square in the family $\mathfrak{B}_{n}$ which intersects $S$; no other members of $\mathfrak{P}_{n}$ intersect $S$. Thus

$$
\int_{\Re_{2}}\left\{f_{n}(u)\right\}^{p} d L(u)=\int_{S} f_{n}(u)^{p} d L(u) \leqq \frac{n^{3 p} \cdot\left(2^{n^{2}}+1\right)^{2}}{n^{2} \cdot 2^{2\left(n^{2}+n\right)}} \leqq \frac{4 n^{3 p}}{2^{2 n}} .
$$

Using this and Minkowski's inequality, it is easily established that

$$
\begin{aligned}
\int_{\Re_{2}}\{g(u)\}^{p} d L(u) & =\lim _{m \rightarrow \infty} \int_{\Re_{2}}\left\{\sum_{k=1}^{m} f_{k}(u)\right\}^{p} d L(u) \\
& \leqq 4\left(\sum_{k=1}^{\infty} \frac{k^{3}}{2^{2 k / p}}\right)^{p}<\infty
\end{aligned}
$$


thus $g \in L^{(p)}$.

\subsection{Corollary. $\Omega^{\prime} \in \mathfrak{B}$.}

For $t \in S$, we let $C_{n, t}$ and $\dot{C}_{n, t}$ denote, respectively, closed squares centered at $t$, with sides parallel to the axes and of length $2^{-n^{2}}$, and $\left(2^{n+1}+3\right) \cdot 2^{-n^{2}}$. We define $D_{n, t}=C_{n, t}+\dot{C}_{n, t} \cdot \sigma \mathfrak{B}_{n}$. We let $M$ denote the number of squares of $\mathfrak{B}_{n}$ which lie entirely within $\dot{C}_{n, t}$, and $N$ the number of squares of $\mathfrak{B}_{n}$ which intersect $\dot{C}_{n, t}$. It is easy to see that

$$
\left(2^{n+1}+1\right)^{2} \leqq M \leqq N \leqq\left(2^{n+1}+3\right)^{2} .
$$

Given $t \in S$, we can always choose $n$ so large that $D_{n, t} \subset S$. For such $n$, taking account of the fact that $L(S)=n^{-2} \cdot 2^{-2\left(n^{2}+n\right)}$ for each $s \in \mathfrak{B}_{n}$,

$$
\begin{aligned}
L\left(D_{n, t}\right) & \leqq L\left(C_{n, t}\right)+\frac{N}{n^{2} \cdot 2^{2\left(n^{2}+n\right)}} \leqq L\left(C_{n, t}\right)+\frac{9}{n^{2}} L\left(C_{n, t}\right) \\
& \leqq 10 L\left(C_{n, t}\right), \\
\Omega^{\prime}\left(D_{n, t}\right) & =\Omega\left(D_{n, t}\right) \geqq \int_{D_{n, t}} f_{n}(u) d L(u) \geqq n^{3} M \cdot n^{-2} \cdot 2^{-2\left(n^{2}+n\right)} \\
& \geqq n L\left(C_{n, t}\right) .
\end{aligned}
$$

Hence

$$
\lim _{n \rightarrow \infty} \frac{\Omega^{\prime}\left(D_{n, t}\right)}{L\left(D_{n, t}\right)} \geqq \lim _{n \rightarrow \infty} \frac{\Omega\left(D_{n, t}\right)}{10 L\left(C_{n, t}\right)}=\infty
$$

and

$$
\lim _{n \rightarrow \infty} \operatorname{diam} D_{n, t} \leqq \lim _{n \rightarrow \infty} \operatorname{diam} \dot{C}_{n, t}=\lim _{n \rightarrow \infty}\left(\frac{2^{n+1}+3}{2^{n 2}}\right) 2^{1 / 2}=0 .
$$

Also, from the next to last inequality of (1), we see that given $\epsilon>0, t \in S$, we have, for suitably large values of $n$,

$$
L\left(D_{n, t}\right) \leqq(1+\epsilon) L\left(C_{n, t}\right) .
$$

We so define $K$ and $H$ that for $t \in S$,

$$
K(t)=\sum_{n=1}^{\infty} S\left(C_{n, t}\right), \quad H(t)=\sum_{n=1}^{\infty} S\left(D_{n, t}\right),
$$

and note from (3) that $K$ and $H$ are blankets with domain $S$. To each set $\beta$ of the form $\beta=D_{n, t}$, there corresponds uniquely a set $\beta^{\prime}=C_{n, t}$, and conversely. Thus there is a one-to-one correspondence between the spreads of $H$ and $K$; henceforth, mention of correspond- 
ing sets will be meant in this sense.

\subsection{ThEOREM. $H$ has the property (I).}

Proof. We select $\epsilon>0$ and choose any subblanket of $H$, say $H_{1}$, with domain $A \subset S$. We use (4) and the fact that $S$ is open to find a subblanket of $H_{1}$, say $H_{2}$, with domain $A$, such that if $\beta$ and $\beta^{\prime}$ are corresponding sets in the spreads of $H_{2}$ and $K$, then

$$
\beta^{\prime} \subset \beta \subset S ; \quad L(\beta) \leqq(1+\epsilon) L\left(\beta^{\prime}\right) .
$$

We so define $K_{2}$ that for $t \in A$,

$$
K_{2}(t)=\sum_{\beta \in H_{2}(t)} S\left(\beta^{\prime}\right),
$$

note that $K_{2}$ is a subblanket of $K$ with domain $A$ whose spread is a family of squares, use the $L$-strength of $K_{2}$ to find a countable disjointed family $\Re$ of its spread which covers $L$-almost all of $A$, let $\mathfrak{S}$ be the corresponding family in the spread of $\mathrm{H}_{2}$, use (5) and the disjointedness of $\Omega$ to see that

$$
\begin{gathered}
L(A-\sigma \mathfrak{S}) \leqq L(A-\sigma \Re)=0, \\
\sum_{\beta \in \mathfrak{W}} L(\beta) \leqq(1+\epsilon) \sum_{\beta \in \mathfrak{S}} L\left(\beta^{\prime}\right)=(1+\epsilon) L(\sigma \Re) \\
\leqq L(\sigma \mathfrak{S})+\epsilon L(S)=L(\sigma \mathfrak{S})+\epsilon,
\end{gathered}
$$

and employ Theorem 4.1 to conclude that

$$
\begin{aligned}
\int_{\sigma \mathfrak{S}}\left(P_{\mathfrak{S}}(u)-1\right) d L(u) & =\int_{\sigma \mathfrak{W}} P_{\mathfrak{S}}(u) d L(u)-L(\sigma \mathfrak{S}) \\
& =\sum_{\beta \in \mathfrak{W}} L(\beta)-L(\sigma \mathfrak{S}) \leqq \epsilon .
\end{aligned}
$$

Hence $H$ has the property (I). Yet from (2) we see that $D\left(\Omega^{\prime}, L, H, t\right)$ $=D(\Omega, L, H, t)=\infty$ for each $t \in S$. Thus, although $\Omega$ was obtained by integrating the function $g$, which is in $L^{(p)}$ for each $p \geqq 1$, the blanket $H$ does not possess covering properties sufficient to insure the differentiability of $\Omega$ with respect to Lebesgue measure.

7. Unions of blankets. We now point out a property of certain sequences of $\phi$-pseudo-strong blankets.

7.1. Definition. We say that $F$ is the union of the family of blankets $\Omega$ if and only if $\Omega \neq 0, f$ is a blanket with domain $A_{f}$ whenever $f \in \Omega$, and $F$ is so defined that for $x \in \mathfrak{S}$,

$$
F(x)=\sum_{f \in \Omega} f(x)
$$


Under these circumstances, it is clear that $F$ is a blanket with domain

$$
\sum_{f \in \Omega} A_{f}
$$

We state the following theorem, the proof being omitted since it is somewhat lengthy, although straightforward.

7.2. THEOREM. If $F$ is the union of such a countable family of blankets 凡 that

(i) $f \in \Omega$ implies $f$ is $\phi$-pseudo-strong;

(ii) the domains of the members of $\AA$ are disjointed;

then $F$ is $\phi$-pseudo-strong.

This theorem insures that the blanket $F$ enjoys the same differentiability properties as the members of $\Omega$ themselves.

University of California, Davis

\section{ON THE SHEFFER A-TYPE OF POLYNOMIALS \\ GENERATED BY $\phi(t) f(x t)$}

WILLIAM N. HUFF AND EARL D. RAINVILLE

1. Introduction. Huff [1] treated polynomial sets $\left\{y_{n}(x)\right\}$ defined by a generating function,

$$
\phi(t) f(x t)=\sum_{n=0}^{\infty} y_{n}(x) t^{n},
$$

in which

$$
\phi(t)=\sum_{n=0}^{\infty} \frac{b_{n} t^{n}}{n !} ; \quad f(x t)=\sum_{n=0}^{\infty} \frac{a_{n}(x t)^{n}}{n !},
$$

with the restriction that neither $b_{0}$ nor any of the $a_{n}$ can be zero. He proved, among other things, a theorem (his 2.1) which states a necessary and sufficient condition that the $\left\{y_{n}(x)\right\}$ above be of Sheffer A-type $k$. For definitions of A-type and a basic treatment of the important Sheffer classifications, see [2].

Starting with Huff's theorem, we shall obtain for $\left\{y_{n}(x)\right\}$ to be of

Presented to the Society, November 23, 1951; received by the editors July 30 , 1951. 